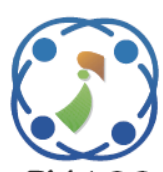

\title{
A Hybrid Model (SVM-LOA) for Epileptic Seizure Detection in Long-Term EEG Records Using Machine Learning Techniques
}

\author{
Mona Abdelbaset Sadek Ali ${ }^{1,2 *}$ \\ Mohamed Abd-Elfattah ${ }^{3}$ \\ ${ }^{I}$ Department of Computer Science, Faculty of Computers and Informatics, Benha University, Egypt \\ ${ }^{2}$ Department of Computer Science, Computer Sciences \& Information Technology, \\ King Faisal University, Saudi Arabia \\ ${ }^{3}$ Department of Information System, Faculty of Computers and Informatics, Benha University, Egypt \\ * Corresponding author’s Email: mona.abdelbaset@fci.bu.edu.eg
}

\begin{abstract}
The aim of this research is to develop a hybrid Model (SVM-LOA) for epileptic seizure detection with support vector machine (SVM) and lion optimization algorithm (LOA) to locate the optimum parameters of support vector machines (SVMs) for classification of Electroencephalogram (EEG) signals. The proposed approach attempts to find the best integration of all available features that offers typical epilepsy detection and gives a better classification rate. Furthermore, the discrete wavelet transform (DWT) has been implemented to divide EEG signals into five combinations. Nonlinear parameters have been calculated and used to be the features to intern the SVM classifier that affects the classification accuracy. We used lion optimization algorithm based approach to optimize the SVM parameters. The overall experimental results shown the LOA-SVM classifiers have $96.78 \%$ for accuracy, but SVM that have obtained $80.05 \%$ for accuracy. Therefore; the SVM-LOA is an efficient model for neuroscientists to detect epileptic seizure in EEG.
\end{abstract}

Keywords: Electroencephalogram (EEG), Epilepsy seizure, Support vector machines (SVMs), Lion optimization algorithm (LOA), Discrete wavelet transform (DWT).

\section{Introduction}

Epilepsy is considered as one of the most popular substantial neurological condition, it is about 60 million people affecting by neurological condition, nearly $1 \%$ of the world population. Every year we have 2.5 million new cases. Nearly $25-30 \%$ of all patients are suffering from incurable epilepsy. Generally, epilepsy patients' death rate is raised by a factor of two to three, with the average annual death percentage of $1 \%$. Epileptic seizures can cause a range of impermanent alterations in perception and behavior [1].

Recognition of seizures in EEG signals can be interesting because of the extreme myogenic artifacts during a seizure. What's more, recording EEG signals for long-term is regularly required for the identification of seizure and the elaboration for surgical interference, principally when finding the epileptogenic foci of the brain and defining the areas to be extracted by operation. Bearing in mind the amount of data, the counting of long-term EEG signals recordings by visual checking is a long time task and overwork. Detection of seizures is extra challenging because a little knowledge is accessible about the mechanism of seizure starting. Subsequently, it deserves to improve influential techniques for automatic seizure discovery and prediction [2, 3]. Recently, warning systems generated by the uncovering of ictal EEG signals patterns gain many interests. As the studies contributory in the finding of seizures by human experts is extremely massive, many efforts have been achieved to develop and enhance automatic seizure detection systems [4].

It is a crucial tool for considerate the brain disease, such as epilepsy [5]. Two states of irregular activities namely interictal (seizure free) and ictal 
(seizure) are detected from EEG signals of epileptic patient [6 - 8]. Considering the huge amount of EEG data that is included in the long-term EEG recording of the patient of a person with epilepsy. Thus, the detection of epileptic activity is a very challenging task and required a detailed examination of the complete length of the EEG data. As a result, the visual inspection of long durations of EEG signals is a conventional method commonly used by experienced neurophysiologists. It leads to timeconsuming. Therefore, a computer-aided detection (CAD) used to overcome these limitations. However, to diagnose and predict epileptic seizures clinically, the brain activities examined over EEG signals that includes epilepsy's markers [9]. Thus, developing an efficient algorithm for automatic seizure detection and prediction is crucial work. Several feature extraction approaches have developed from diverse focusing points. These approaches include frequency domain, time domain, and time frequency domain, additional to nonlinear methods. As a result, the time-frequency study has explored, whose significant idea is to stratify diverse decomposition methods, with the

Wavelet transform (WT) [10]. The other challenge need to consider is selecting a resourceful classifier to conduct the seizure discovery. The generally utilized classifiers in seizure discovery e.g. natural nervous system (ANNs), Decision tree (DT) and SVM. Artificial neural-networks are a system that processes the information as a natural nervous system (ANNs). It has been functional to differentiate the EEG. Decision tree (DT), designed by a collection of nodes produced from an arrangement of questions along a pathway from root to leaf. DT has used to be the classifier. In the meantime, SVM targets to discover the prime separating hyper-plane on the base of the statistical learning theory. It is one of the greatest common machine learning tools in pattern recognition field. In addition, the merits of SVMs are; 1) classify two diverse sets of notes into their related class, 2) handling the nonlinear and high-dimensional data [11]. As a promising technology, (ELM) extreme learning machine attracts recently the considerations from many scientists due to its effectiveness in regression and classification problems, including seizure discovery in [12].

This research proposed an efficient developed algorithm for automatic seizure detection and classification for epileptic seizure detection in longterm EEG. This article focuses on; DWT that has been utilized to decompose EEG signals into five sub-band components, LOA has been utilized to develop a LOA-SVM classification approach for
SVM parameters optimization and feature selection that improves the classification rate. Additionally, this approach is capable of discriminating of epileptic and non-epileptic accurately. The article organized as follows: in Section 2, a literature review is illustrated.

Materials and methods introduced in Section 3. Then, the proposed classification approach discussed in Section 4. In Section 5, the results obtained and details about the evaluation data provided. Finally, the paper close with the conclusions and future work in Section 6.

\section{Literature review}

Various algorithms have been introduced in the literature to examine EEG Signals in order to determine the presence or absence of ongoing seizures. Consequently, many types of the classifier utilized in the previous researches work such as ANN, Fuzzy system, and SVM of different biometric signals presented in Table 1. This section introduces some reviews for ECG signals classification ranging from 2013 to 2017.

\section{Materials and methods}

This section presents the EEG data acquisition, pre-processing and feature extraction using DWT, feature selection and classification using LOA optimized SVM.

\subsection{Electroencephalogram (EEG) Signals}

The EEG signals ordinarily decomposed of five EEG sub-bands: delta, theta, alpha, beta, and gamma. Alpha waves are consistent within every region of the brain has the distinctive of alpha harmony but particularly it is registered from the occipital and parietal regions. It fluctuates from an adult in the awake and stress-free state with closed eyes. Beta waves are infrequent and foremost noted from temporal and frontal lobe. It fluctuates from 'during the deep sleep', 'mental action' and 'connected with memorizing'. Delta waves are consistent and it fluctuates from the kids in a sleep state, dozy adult and emotional distress occipital lobe. Theta waves are slow and it fluctuates from adult and normal sleep harmony. Gamma waves are the rapid brainwave frequency. Table 2 shows the frequency range and amplitude for each wave [13].

\subsection{Discrete wavelet transform (DWT)}

Obtaining the accurate feature combinations considered a challenging task. Actually, the number 
Table 1. Summary of related work

\begin{tabular}{|c|c|c|c|}
\hline Studies & Classifier & Measure & Remark \\
\hline [19] & SVM & Ac: $99.54 \%$ & $\begin{array}{l}\text { Detected the main points at different levels in EEG signals using the } \\
\text { difference pyramid of Gaussian (DoG) filtered signals. }\end{array}$ \\
\hline [20] & LS-SVM & Sens: $100 \%$ & $\begin{array}{l}\text { Proposed automatic algorithm to detect epileptic seizures based on Fractal } \\
\text { Dimension (FD) and Analytic Time-frequency Flexible Wavelet Transform } \\
\text { (ATFFWT) }\end{array}$ \\
\hline [21] & SVM & Ac: $98.6 \%$ & Calculated the entropy of the EEG signal in many frequency-bands. \\
\hline [22] & MLPNN & Ac: $99.3 \%$ & $\begin{array}{l}\text { Hybridized three-band filter banks and multi-layer perceptron neural } \\
\text { network (MLPNN) for epileptic seizure classification. }\end{array}$ \\
\hline [23] & $\mathrm{K}-\mathrm{NN}$ & Sens: $88 \%$ & $\begin{array}{l}\text { Introduced a supervised ML approach that classifies seizure and non- } \\
\text { seizure. }\end{array}$ \\
\hline [24] & $\begin{array}{l}\text { SVM and } \\
\text { PSO }\end{array}$ & Ac: $89.7 \%$ & $\begin{array}{l}\text { Optimization classifier was evaluated to measure the performance with } \\
\text { motor imagery EEG signals. }\end{array}$ \\
\hline [25] & $\mathrm{K}-\mathrm{NN}$ & Sens: $93 \%$ & Utilized the EEG signals to classify seizure and non-seizure. \\
\hline [26] & $\mathrm{K}-\mathrm{NN}$ & Ac: $98.33 \%$ & Utilized Gabor filters for processing and K-NN classifier. \\
\hline [27] & SVM & Ac: $87 \%$ & Exploring the various visual object stimuli can trigger detection. \\
\hline [28] & $\begin{array}{l}\text { SVM and } \\
\text { ANN-MLP }\end{array}$ & Ac: $89.2 \%$ & SVM-RBF and ANN-MLP are proposed \\
\hline [29] & SVM & Ac: $95.33 \%$ & $\begin{array}{l}\text { Utilize the Fractional linear prediction (FLP) to model ictal and seizure-free } \\
\text { EEG. }\end{array}$ \\
\hline [13] & $\mathrm{NN}$ & Sens: $8.98 \%$ & Proposed ensemble in the classifications of seizure and non-seizure \\
\hline [31] & SVM & Ac: $82.14 \%$ & $\begin{array}{l}\text { Providing an effective embedded approach for feature election and linear } \\
\text { discrimination. }\end{array}$ \\
\hline [32] & SVM & Sens: $73 \%$ & 22 linear univariate feature space extracted from six EEG recordings. \\
\hline
\end{tabular}

Table 2. ECG Waves [13]

\begin{tabular}{|c|c|c|}
\hline Wave & Frequency range & Amplitude \\
\hline Delta band & $0.1-4 \mathrm{~Hz}$ & High \\
\hline Theta band & $4-8 \mathrm{~Hz}$ & $\begin{array}{l}\text { Low- } \\
\text { medium }\end{array}$ \\
\hline Alpha band & $8-15 \mathrm{~Hz}$ & Low \\
\hline Beta band & $15-30 \mathrm{~Hz}$ & Very low \\
\hline Gamma band & $30-100 \mathrm{~Hz}$ & Smallest \\
\hline
\end{tabular}

of features located in the literature considered very big. Moreover, it required a lot of computational cost. So, selecting the features that are related to a certain kind of illness. The selected features are specified from the review and are aggregated together to create a single feature vector. DWT is mainly valuable for the investigation of transients, a periodic and other non-fixed signal features. Therefore it is so valuable in the handling of EEG signals [3], "In order to decompose the ECG signal into time frequency components, let us consider two variables ' $a$ ' and ' $b$ ' named as scale and translation parameters respectively. The scale ' $a$ ' defines the amount of dilation (i.e. either stretching or contraction) and the translation ' $b$ ' defines the quantity of shift of the basis function for the time frequency transformation. Such a basis function at scale ' $a$ ' and translation ' $b$ ' is defined by the following wavelet Eq. (1) [38].

$$
\psi_{\mathrm{m}, \mathrm{n}}(\mathrm{t})=\frac{1}{\sqrt{\mathrm{a}_{0}^{\mathrm{m}}}} \psi\left(\frac{\mathrm{t}-\mathrm{n} \mathrm{b}_{0}}{\mathrm{a}_{0}^{\mathrm{m}}}\right)
$$

The mother wavelet, basis function, is defined at unit scale and zero translation, where its shape must be similar to ECG signal morphology. The parameters a and b are sampled on a dyadic grid, and such a wavelet transform is called as dyadic grid wavelet transform whose wavelet equation leads to

$$
\psi_{\mathrm{m}, \mathrm{n}}(\mathrm{t})=2^{\frac{-\mathrm{m}}{2}} \psi\left(2^{-\mathrm{m}} \mathrm{t}-\mathrm{n}\right)
$$

These discrete dyadic grid wavelets are generally orthonormal having unit magnitude and being perpendicular to each other. Using the dyadic grid wavelet transform of eqn. 2, the discrete wavelet transform (DWT) is given by

$$
\mathrm{T}_{\mathrm{m}, \mathrm{n}}=\int_{-\infty}^{\infty} \mathrm{X}(\mathrm{t}) \psi_{\mathrm{m}, \mathrm{n}}(\mathrm{t}) \mathrm{dt}
$$

The DWT of a given ECG signal is obtained as the inner product between the basis wavelet and the 
signal itself as shown in eqn. (4). These basis functions are orthogonal, while the inner product between the signal and an orthogonal basis function is performed, the information in the signal will be present in the wavelet transform only at that particular ' $a$ ' and ' $b$ ' choices and will not be repeated anywhere else in time frequency domain. Hence wavelet transforms of given ECG signal gives orthogonal features having compact supported basis for the frame" [38]. Where $T_{m, n}$ is recognized as the wavelet coefficient at scale and location indices $(m, n)$. The chosen of the wavelet and levels of decomposition is critical to examining of EEG signals [14].

\subsection{Lion optimization algorithm (LOA)}

Feature selection techniques of datasets provide a procedure to distinguish between features and removing irrelevant features. The major purposes of feature selection are reducing data dimensions and improving prediction performance. Moreover, there are several heuristic procedures that copying the hunting behaviour of physical and biological systems in nature. So they have been planned as approaches for global optimizations of the problems. Maziar et al., have proposed an optimization algorithm based on lion's behaviour, namely Lion Optimization Algorithm (LOA) [15]. In LOA, primary population established by a set of arbitrarily formed solutions named Lions. Some of primary population ' $\% \mathrm{~N}$ ' are chosen as nomad lions. The rest population is arbitrarily divided into $\mathrm{P}$ subsets named pride. ' $\mathrm{S} \%$ ' is considered as female and the rest are considered to be male of the pride's members or vice versa. For every lion, the best acquired result in handed repetitions is titled bestvisited position, and through the optimization procedure is updated progressively. In LOA, a pride province is an extent that contains every member best-visited point. In every pride, several females that are randomly nominated go hunting. Hunters go to the victim to surround and grab it. The remainder of females go to diverse locations of the province. Male lions in the pride, roam in the province. Females in pride mate with one or some habitat males. Every fresh males are removed from their parental pride and adapt to be nomad when they become adult and, with less power than habitat males. Also, a nomad lion travels arbitrarily in the search space to find a good place. The habitat male is exclude of the pride if the powerful nomad male interrupts the habitat male by the nomad lion. The nomad male converts to the habitat lion. In the evolution, some habitat females immigrate from one pride to another or switch their lifestyles and convert a nomad and vice versa, some nomad female lions combine pride. Many factors result the death and killing of powerless lion, lack of food and competition. Overhead procedure remains till the interception state is satisfied and for more details about LOA [15].

\subsection{Support vector machines (SVM)}

Vapnik as a solution for function approximation problem introduced first contribution of SVMs by a machine learning technique [16]. SVM is a popular classifier that utilizes kernels to build linear classification boundaries in higher dimensional space. SVM classifier is simple to implement and moderate in computational requirement. Besides, it is straightforward to calculate classification accuracy. The core idea of SVM is to create a hyperplane as a decision surface to maximize the separation between positive and negative models [17, 18]. SVM is a convergent application of structural risk reduction. The SVM provides labeled training data;

$$
\begin{aligned}
D= & \left\{\left(x_{i}, y_{i}\right)\right\}_{i=1}^{l}, x_{i} \in X \subset R^{d}, \\
& y_{i} \in Y=\{-1,+1\}
\end{aligned}
$$

Creates a maximal margin linear classifier in a high dimensional feature space $\phi(x)$ defined by a positive definite kernel function $k\left(x, x^{\prime}\right)$ allocating an inner product in the feature space;

$$
\phi(x) . \phi\left(x^{\prime}\right)=k\left(x, x^{\prime}\right)
$$

A popular kernel is the Gaussian radial basis function (RBF);

$$
k\left(x, x^{\prime}\right)=e^{-\left\|x-x^{\prime}\right\|^{2} / 2 \sigma^{2}}
$$

The function implemented by an SVM is provided by;

$$
f(x)=\left\{\sum_{i=1}^{l} \alpha_{i} y_{i} k\left(x_{i}, x\right)\right\}-b
$$

To find the optimal coefficients of this expansion, it is adequate to maximize the function;

$$
w(\alpha)=\sum_{i=1}^{l} \alpha_{i}-\frac{1}{2} \sum_{i, j=1}^{l} y_{i} y_{j} \alpha_{i} \alpha_{j} k\left(x_{i}, x_{j}\right.
$$

The major goal of SVM is to minimize the expectation of the output of pattern error. SVM map is a given set of binary labelled of each training 


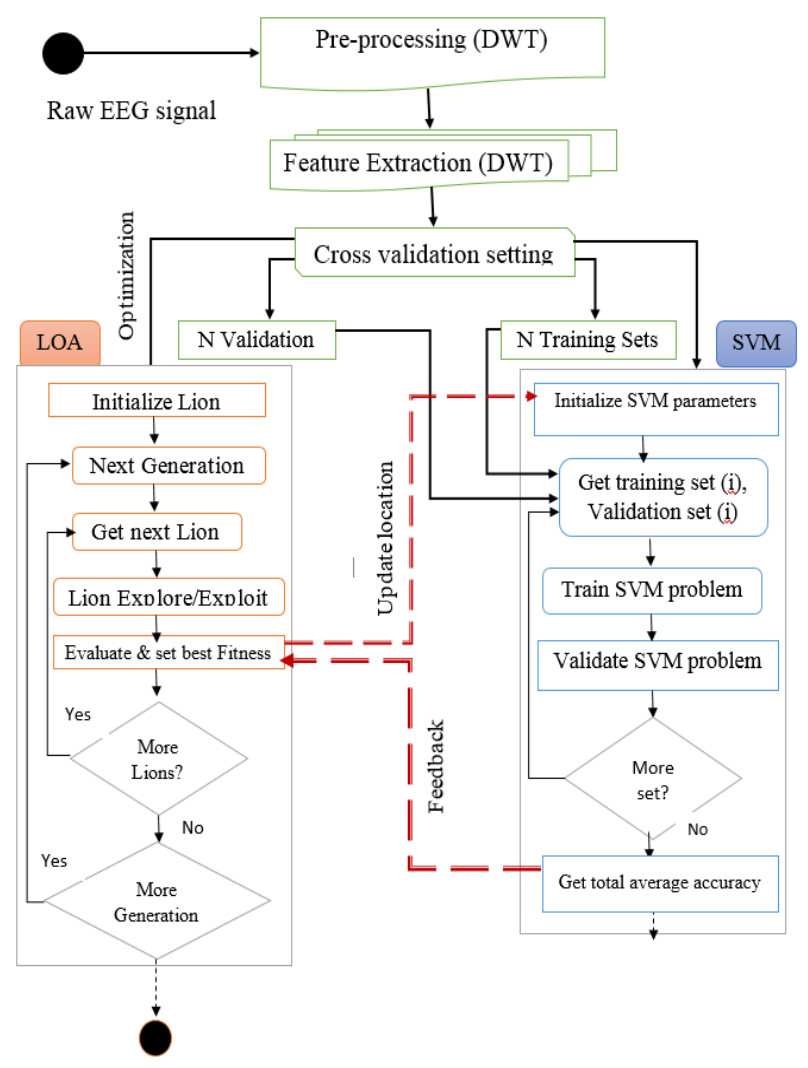

Figure. 1 The general framework of the proposed classification approach for EEG signals

pattern to a high dimensional feature space and separates the two classes of the pattern is obtainable with a maximum margin of hyper-plane.

\section{Proposed LOA-SVM classification approach}

In this section, LOA-SVM classification approach proposed for the EEG signal described. At this approach, the SVM classifier accuracy optimized by automatically solving the SVM model selection by approximating the best values of the kernel parameters. To accomplish this, the approach is resulting from an optimization framework based on LOA. In order to implement the suggested approach, the RBF kernel function has utilized for the SVM classifier. Once the RBF kernel is nominated, the parameters $\mathrm{C}$ and need to be optimized using LOA. Due to the good performances of RBF kernel, it is typically familiar with the non-linear SVM classifier. Classification accuracy is a criterion used to design a fitness function. Thus, for the lion with high classification accuracy provides a high fitness value. The fitness has reserved as the classification accuracy. As a result, LOA-SVM approach has utilized as a powerful tool for EEG signal classification. The suggested classification method, involves four phases: 1) Pre-processing used to get rid of the noises from the EEG signals. 2) Feature extraction used to extract the EEG signal features from decomposed signal. 3) Feature selection choose the important features from the extracted features and 4) Classification phases in this phase, the selected features had given as input to the classifier. The classification phase mainly has used to analyse the EEG signals and it classifies the epileptic EEG into normal or abnormal, as seen in Fig. 1.

The proposed approach aims to obtain the optimal parameter settings for the SVM classifier as well as selecting best features related to the problem. This achieved by employing LOA optimization algorithm to fine-tune classification process and find best results. It also applies radial basis kernel function (RBF) to guarantee best output. Algorithm 1 shows the algorithmic framework of LOA-SVM classifier. In step 1 and 2, processing of EEG signals goes through two steps. Firstly, it starts by the decomposition of EEG signals into different frequency bands using DWT. In the second step, resultant sub-bands decomposed EEG signals undergo a statistical feature extraction phase to get better accuracy for analysis of neurogenic disorders.

\begin{tabular}{l} 
Algorithm 1: LOA-SVM Algorithm. \\
\hline 1: Input: Training Sets (Folds) (T 1; T $2::: \mathrm{T} \mathrm{n})$ \\
2: Input: Validation Sets (Folds) (V1; V2 : : Vn) \\
3: Output: Classification Accuracy \\
4: Initialization: Population locations (SVM, \\
Kernel parameters and Selected Features), all \\
members of brides and no-mad lions and \\
generate a random population of Lions. \\
5: Evaluate population fitness (g) (Alg. 2, Eq. (9)). \\
6: for each bride do \\
7: Male and female nomad move randomly. \\
8: Ratio of nomad females with the best nomad \\
9: $\quad$ males. \\
10:end for \\
11: for each bride do \\
12: Randomly female immigrate from pride = \\
13: $\quad$ Eachad. \\
14: The bender of nomales are distributed to the prides. \\
15: end for \\
16: Evaluate population fitness (g) (Alg. 2, Eq. (9)). \\
17:Find Best lions with Highest Accuracy. \\
18:Exit
\end{tabular}


Table 3. Summary of dataset

\begin{tabular}{|c|c|c|c|c|c|}
\hline Settings & A & B & C & D & E \\
\hline Subjects & 5 healthy & 5 healthy & $\begin{array}{c}\text { 5 epileptic } \\
\text { patients }\end{array}$ & 5 epileptic patients & 5 epileptic patients \\
\hline $\begin{array}{c}\text { Electrode } \\
\text { type }\end{array}$ & surface & surface & intracranial & Intracranial & Intracranial \\
\hline $\begin{array}{c}\text { Electrode } \\
\text { placement }\end{array}$ & $\begin{array}{c}\text { International } \\
10-20 \text { system }\end{array}$ & $\begin{array}{c}\text { International 10-20 } \\
\text { system }\end{array}$ & $\begin{array}{c}\text { Epileptogenic } \\
\text { zone }\end{array}$ & $\begin{array}{c}\text { Hippocampal } \\
\text { formation }\end{array}$ & Epileptogenic zone \\
$\begin{array}{c}\text { Patient's } \\
\text { state }\end{array}$ & $\begin{array}{c}\text { Awake, eyes } \\
\text { open }\end{array}$ & Awake, eyes closed & $\begin{array}{c}\text { Seizure-free } \\
\text { (Interictal) }\end{array}$ & $\begin{array}{c}\text { Seizure-free } \\
\text { (Interictal) }\end{array}$ & $\begin{array}{c}\text { Seizure activity } \\
\text { (Ictal) }\end{array}$ \\
\hline $\begin{array}{c}\text { Number } \\
\text { of epochs }\end{array}$ & 100 & 100 & 100 & 100 & 100 \\
\hline $\begin{array}{c}\text { Epoch } \\
\text { duration }\end{array}$ & 23.6 & 23.6 & 23.6 & 23.6 & 23.6 \\
\hline
\end{tabular}

\subsection{Future selection}

Besides SVM parameter optimization, in step 3, Feature selection is utilized to recognize a strong predictive subset of fields within a database and reduce the number of fields displayed on calculation process. The choice of feature affects many aspects of classification, containing the validity of classification, the time required to learn, the number of examples required to learn, and the cost-related features. In a precise application problem like EEG signals, features have different level of their important. For getting optimal performance can be accomplished by discarding some features. Thus, the noise is simply eliminated, along with eliminating irrelevant and redundant data during the improvement of the discriminating power of the data by selecting feature. The optimization stage is triggered where the algorithms performs repeated feature and SVM parameters optimization.

\subsection{Fitness function}

In this paper, classification accuracy has been selected as the solution qualifier through search process. Classification accuracy lies in the range [0:1], each Lion reflects a number of accuracies depends on cross validation strategy. In this paper, each Lions reflects three accuracy values for each fold in a 3-fold cross validation strategy, all accuracy values for all folds are averaged to return fitness value to the search algorithm as shown in the following Eq. (9).

$$
f(i, j)=\frac{\sum_{k=1}^{n} A c c_{i, j, k}}{n}
$$

Where $f(i, j)$ is the fitness value for Lion $\mathrm{i}$ in iteration $\mathrm{j}$. $\mathrm{n}$ represents the number of folds selected for cross validation. $A c c_{i, j, k}$ is the resultant accuracy of evaluation for Lion $\mathrm{i}$ in iteration $\mathrm{j}$ and for the data fold $k$.

\section{Experimental results and discussion}

\subsection{EEG signals pre-processing}

The data used in this study were captured by the standard available data in the Epilepsy Department, University of Bonn [33]. This data set contains five groups (referred to as A, B, C, D and E), each containing 100 single channel EEG channels of 23.6 seconds, with a sampling rate of $173.6 \mathrm{~Hz}$. Where each data slice contains $=4097$ data points accumulated at intervals of $1 / 173.61$ of $1 \mathrm{~s}$. These segments are selected from continuous multichannel EEG recordings after a visual investigation for artifacts, e.g., due to muscle activity or eye movements.

The datasets A and B included segments taken from surface EEG recordings that were applied on five healthy volunteers using a unified electrode placement scheme. The volunteers stressed-free in an awake state with eyes open (A) and closed eyes (B), correspondingly. The data sets C, D, and E are recorded from the epileptic subjects through intracranial electrodes for interictal and ictal epileptic activities. All focuses had done complete seizure control after the eradication of one of the hippocampal formations, which was carefully analyzed to be an epileptic region. Sections in set D were recorded from the epileptogenic zone and those in set $\mathrm{C}$ from the hippocampal formation of the adverse hemisphere of the brain. While sets $\mathrm{C}$ and $\mathrm{D}$ comprised only activity estimated during seizure free intervals, set E only included epileptic seizure activity. A summary of the datasets is shown in Table 3.

All EEG signals had been recorded with the same 128-channel amplifier system, using a 
common reference average. After analog 12-bit conversion, the data was continuously on computer system disk to obtain data at a sampling rate of $173.61 \mathrm{~Hz}$. They have the spectral bandwidth of the acquisition system, which varies from 0.5 to $85 \mathrm{~Hz}$.

\subsection{Performance evaluation}

The datasets are divided as follows; 1) the training data, 2) the validation data and 3) the test data, in order to estimate a classifier performance. Training set is used for learning (training) the classifier. The validation data task is to select best classifier parameter setting and adjust its parameters. Finally, the test data is used to estimate the final model error rate. The three sets are selected as separate data sets or independent portions of original data. Validation and training sets must be independent to get best results in parameter selection stage. Also test set must be different from other sets to get consistent evaluation of true error rate. In order to divide the dataset, cross-validation (CV) imperative statistical techniques are always applied. Cross validation requires selection of a fixed number of folds, each represents a separate or overlapped portion of the data. k-fold cross validation in general is aimed to eliminate the bias of classifier and boosts the model ability to generalize, which is related to random sampling of the training and test sets [34]. A known method for error rate prediction is applying cross validation in 3 -folds of data. Data divided into three distinct partitions of equal sizes, each partition is separately used for testing and other partitions are used for training the classifier.

\subsection{Feature extraction using DWT}

Extracting the main features of Epileptic EEG has been extracted through two phases. In the first phase, discrete wavelet transform is computed to decompose the EEG signal into numerous subsignals within diverse frequency bands. Choosing the number of decomposition levels and convenient wavelet function are also essential for EEG signal examination with DWT. The number of decomposition levels is selected 4, which is suggested. Also, the function of carefully selected wavelets is the Daubechies of system 4, which is also confirmed to be the best appropriate wavelet function for signal analysis of EGG epileptic [22]. Table 4 shows frequency bands that respond to 4 level decomposition with the sampling frequency of 173.6 on the EEG signal.
Table 4. Frequency bands of EEG signal with 4-level DWT decomposition

\begin{tabular}{c|c|c}
\hline $\begin{array}{c}\text { Decomposition } \\
\text { level }\end{array}$ & $\begin{array}{c}\text { Sub-band } \\
\text { signal }\end{array}$ & $\begin{array}{c}\text { Frequency band } \\
(\mathbf{H z})\end{array}$ \\
\hline 1 & D1(gamma) & $30-60$ \\
2 & D2 (beta) & $15-30$ \\
3 & D3 (alpha) & $8-15$ \\
4 & D4 (theta) & $4-8$ \\
5 & A4 (delta) & $0-4$ \\
\hline
\end{tabular}

These Daubechies wavelet coefficients were calculated and examined by utilizing MATLAB. The five different sub-signals are mentioned in Table 4. In order to build the core database, ten conventional features are extracted from the approximation and detail coefficients of all subbands of the entire 500 EEG epochs of five data sets A-E. In this paper, DWT technique has been utilized to evolve ten features from EEG signal in which can be utilized to categorize epileptic seizure. From personal point of view, the number of features extracted is considered the best when compared to other studies.

\subsection{Feature selection and classification based on LOA-SVM classification approach}

The accurate selection of appropriate features from EEG signals can support to design a classifier with better popularization performance. The selection of a subset of features increases classification accuracy. Consequently, a feature selection is an essential task in order to solve the dimensionality obstacle. Essentially, when datasets are very large, it is crucial to study individual features. Furthermore, features reduction will assist the classifier to learn a more powerful solution and accomplish a better popularization performance. Because unrelated feature components are discarded by the optimum sub-space projection. In this paper, LOA is utilized for feature selection and to optimize the SVM parameters.

\subsection{Parameters settings and optimization}

Table 5, summarize the cross-validation, SVM parameter, and LOA parameter settings. In step 3, setting the parameters of SVM has an essential effect on its regression correctness. Incorrect classification results from using unsuitable parameter. To get SVM optimal classification accuracy requires searching for best parameters setting. The parameters should be optimized include the error penalty parameter $\mathrm{C}$ and the kernel function parameters such as the RBF kernel parameter $\gamma$. 
Table 5. Parameter settings

\begin{tabular}{l|l}
\hline SVM & LOA-SVM \\
\hline Kernel: Radial Basis & Number of pride : 8 \\
Penalty Optimization & Percent of nomad lions : 0.4 \\
Range:[1, 1000] & Roaming percent : 0.4 \\
Gamma Optimization & Mutate probability : 0.4 \\
Range: $[0,1000]$ & Sex rate : 0.8 \\
Data Scaling Factor: & Mating probability : 0.3 \\
$\quad[-1,1]$ & Immigrate rate $: 0.4$ \\
\hline
\end{tabular}

\subsection{Results and discussion}

In order to evaluate the performance of the proposed approach, input EEG data is split into training and test sets, then k-fold cross-validation (leave one out) was applied subsequently [34]. Each fold approximately includes the same amount of epileptic and non-epileptic classes; this is to make classification more valid. In this paper, the value of $\mathrm{k}$ is set to three; hence, EEG dataset was divided into 3 distinct parts. Two parts are used to train the classifier, while the third part (which is unseen by the classifier) is used to test classification. Here, the proposed approach implemented the one against all multi-class SVM system to fine-tune the SVM parameters and feature set selection, parameter optimization setting for SVM have applied with radial basis kernel function (RBF). RBF is the most popular kernel functions and the _ parameter varied between ( 1 and 100), is defined by:

It clear from the experimental results, LOASVM shows an improved performance with respect to all measures compared to SVM results. Figures 2 and 3 , shows a graphical comparison of all output accuracy measures for SVM and LOA-SVM separately. The proposed classification approach targets optimal classification results using a guided and heuristic search paradigm of the SVM and Kernel parameters' values instead of older empirical tuning approaches. LOA-SVM classifiers were applied to the available training dataset. Comparing results, it is shown that best results are obtained by LOA-SVM classifier applying RBF function. Best accuracy was achieved $96.78 \%$ compared to SVM classifiers.

Even though some studies have been performed for epileptic EEG, the swarm optimization algorithms was applied as an improvement technique to get the optimal parameters of the classifier. Furthermore, the feasibility of swarm optimization algorithms as a feature selector for automatic seizure detection has not been examined so far, on the other hand, the previous studies was applied DWT to extract some feature (frequency or time domain or entropy) but not all the features.

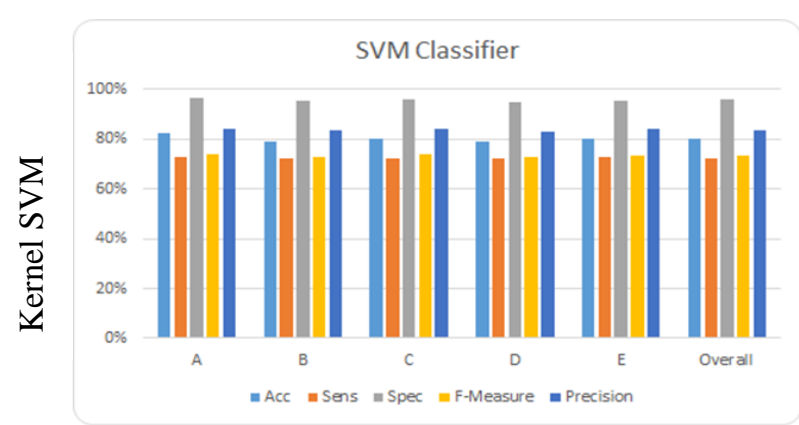

Figure. 2 Visual comparison between the results were obtained by SVM classifiers

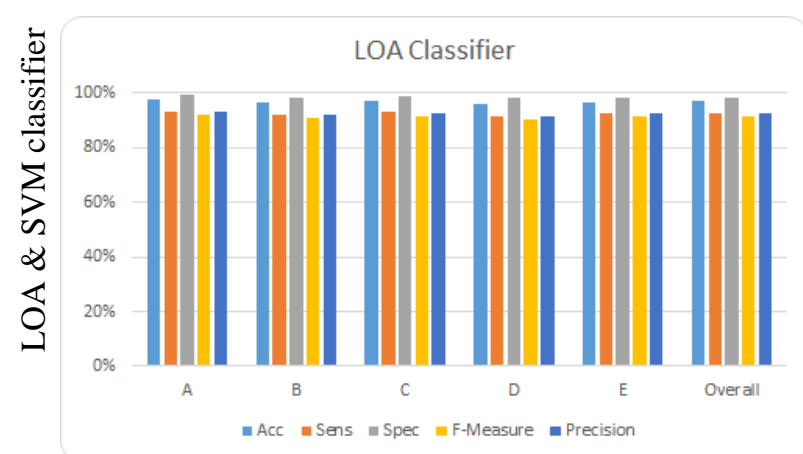

Figure. 3 Visual comparison between the results were obtained by LOA-SVM classifiers

Moreover, in this paper, the DWT has been utilized to extract ten features. And LOA has been applied as an optimization technique to select the relevant feature besides SVM parameter optimization to obtain accurate performance accuracy. Our findings with regards to applied DWT to extract ten distinct feature and LOA to select the relevant feature besides parameter optimization have been achieved higher performance measurements in all matrices. In addition, for all parameter combinations, dataset $\mathrm{A}$ achieves the best accuracy, sensitivity, specificity, FMeasure and precision.

\subsection{Comparison with existing techniques}

A straight assessment of the results with the earlier literature in EEG epileptic detection was challenging due to the diversity of EEG datasets, wavelet types, decomposition levels, participant's variability, and the cognitive tasks used. As a result, a brief comparison with the earlier related studies is introduced in Table 6. Only the methods using the same data for the same cases are incorporated because a comparison between the results is realistic. Consequently, total classification accuracy result of the proposed classification approach provides 96.78\% accuracy, is the best when compared to other studies using similar classifier and was applied 
Table 6. Comparison of classification accuracy obtained

\begin{tabular}{c|c|c}
\hline Studies & Approach & Accuracy \\
\hline$[30]$ & GA-SVM & $95.8 \%$ \\
{$[35]$} & PSO-SVM & $95.2 \%$ \\
{$[36]$} & SVM & $86.10 \%$ \\
{$[37]$} & ANN & $95 \%$ \\
{$[29]$} & SVM & $95.33 \%$ \\
{$[24]$} & PSO-SVM & $89.7 \%$ \\
Proposed & LOA-SVM & $96.78 \%$ \\
\hline
\end{tabular}

swarm optimization algorithms to optimize classification results of EEG signals for epileptic detection. Comparison of results and methods with other studies are presented in Table 6.

\section{Conclusion}

Epilepsy is one of the most prevalent neurological conditions and epileptic seizures can be predicted by analysis of EEG recordings. This paper develops classification approach based on LOA and SVM classifier for epilepsy detection in EEG signals. In this research, the challenge of classifying multichannel EEG data by many time and frequency domain features into epileptic and non-epileptic are presented. Hence, only the most considerable features were utilized for testing and training the classifier model SVM. Overall, the classification, evaluation and evaluation of classification models were used using subsets of features and revealed the most important features of the classification function. Finally, the use of LOA for feature selection increased significantly the performance of SVM classification. As the result demonstrates the effectiveness of using LOA and SVM classifiers for seizure detection in EEG signals, the proposed classification approach provide high classification results i.e., SVM (accuracy $=80.05 \%$, sensitivity $=$ $72.47 \%$, specificity $=95.66 \%$, F-measure $=73.37 \%$ and precision $=83.72 \%$ ) and LOA-SVM classifier (accuracy $=96.78 \%$, sensitivity $=92.49 \%$, specificity $=98.45 \%$, F-measure $=91.31 \%$ and precision $=92.28 \%$ ). As future work, the proposed approach can be applied to a more wide range of pattern recognition problems, which are important to humans, such as the Alzheimer's, diseases detection and diagnosis. In addition, hybridization of machine learning classifiers such as the recent nonparallel support vector machine (NPSVM) and spike neural networks into a single algorithm is a line of research under investigation and a worthwhile research point.

\section{References}

[1] A. V. Devel, K. Cuppens, B. Bonroy, M. Milosevic, K. Jansen, S. V. Huffel, B. Vanrumste, P. Cras, L. Lagae, and B. Ceulemans, "Non-EEG seizure detection systems and potential SUDEP prevention: State of the art: Review and update", Seizure, Vol. 141, pp.141-153, 2016.

[2] C. B. Josephson, S. Sandy, N. Jette, T. T. Sajobi, D. Marshall, and S. Wiebe, "A systematic review of clinical decision rules for epilepsy", Epilepsy \& Behavior, Vol.57, pp.6976, 2016.

[3] H. Asmaa, E. H. Houssein, A. E. Hassanien, and A. Fahmy, "A Hybrid EEG Signals Classification Approach Based on Grey Wolf Optimizer Enhanced SVMs for Epileptic Detection", In: Proc. of International Conference on Advanced Intelligent Systems and Informatics, pp.108-117,2017.

[4] M. Musselman and D. Djurdjanovic, "Timefrequency distributions in the classification of epilepsy from EEG signals", Expert Systems with Applications, Vol.39, pp.11413-11422, 2012.

[5] L. Guo, D. Rivero, J. Dorado, C. R. Munteanu, and A. Pazos, "Automatic feature extraction using genetic programming: An application to epileptic EEG classification", Expert Systems with Applications, Vol. 38, pp.10425-10436, 2011.

[6] U. R. Acharya, H. Fujita, V. K. Sudarshan, S. Bhat, and J. E. Koh, "Application of entropies for automated diagnosis of epilepsy using EEG signals: a review", Knowledge-Based Systems, Vol. 88, pp. 85-96, 2015.

[7] E. B. Assi, D. K. Nguyen, S. Rihana, and M. Sawan, "Towards accurate prediction of epileptic seizures: A review", Biomedical Signal Processing and Control, Vol.34, pp.144-157, 2017.

[8] H. Asmaa, E. H. Houssein, A. E. Hassanien, and A. A. Fahmy, "Feature extraction of epilepsy EEG using discrete wavelet transform", In: Proc. of the 12th International Computer Engineering Conference, pp.190195, 2016.

[9] S. Patidar and T. Panigrahi, "Detection of epileptic seizure using Kraskov entropy applied on tunable-Q wavelet transform of EEG signals", Biomedical Signal Processing and Control, Vol. 34, pp.74-80, 2017.

[10] L. Guo, D. Rivero, J. Dorado, J. R. Rabunal, and A. Pazos, "Automatic epileptic seizure 
detection in EEGs based on line length feature and artificial neural networks", Journal of Neuroscience Methods, Vol.191, pp.101-109, 2010.

[11] C. Cortes and V. Vapnik, "Support-vector networks", Machine Learning, Vol.20, pp.273297, 1995.

[12] J. L. Song, W. Hu, and R. Zhang, "Automated detection of epileptic EEGs using a novel fusion feature and extreme learning machine", Neurocomputing, Vol. 175, pp.383-391, 2016.

[13] S. Dehuri, A. K. Jagadev, and S.B. Cho, "Epileptic seizure identification from electroencephalography signal using DERBFNs ensemble", Procedia Computer Science, Vol. 23, pp.84-95, 2013.

[14] B. Priyadarshini, R. K. Ranjan, and R. Arya, "Determining ECG characteristics using wavelet transforms", International Journal of Engineering Research and Technology, Vol.1, pp.2278-018, 2012.

[15] M. Yazdani and F. Jolai, "Lion Optimization Algorithm (LOA): A nature- inspired metaheuristic algorithm", Journal of Computational Design and Engineering, Vol. 3, pp.24-36, 2016.

[16] V. N. Vapnik, "the Nature of Statistical Learning Theory", Springer-Verlag New York, Inc., New York, USA, 1995.

[17] M. Sabeti, R. A. Boostani, S. D. Katebi, and G. W. Price, "Selection of relevant features for EEG signal classification of schizophrenic patients", Biomedical Signal Processing and Control, Vol.2, pp.122-134, 2007.

[18] Q. Wu and D. X. Zhou, "Analysis of support vector machine classification", Journal of Computational Analysis \& Applications, Vol. 8, 2006.

[19] A. K. Tiwari, R. B. Pachori, V. Kanhangad, and B. K. Panigrahi, "Automated Diagnosis of Epilepsy Using Key-Point-Based Local Binary Pattern of EEG Signals", IEEE Journal of Biomedical and Health Informatics, Vol. 21, No.4, pp.888-896, 2017.

[20] M. Sharma, R. B. Pachori, and U. Rajendra Acharya, "A new approach to characterize epileptic seizures using analytic time-frequency flexible wavelet transform and fractal dimension", Pattern Recognition Letters, Vol. 94, pp. 172-179, 2017.

[21] A. Bhattacharyya, R. B. Pachori, A. Upadhyay, and U. R. Acharya, "Tunable-Q Wavelet Transform Based Multiscale Entropy Measure for Automated Classification of Epileptic EEG
Signals", Applied Sciences, Vol. 7, pp. 385, 2017.

[22] D. Bhati, M. Sharma, R. B. Pachori, and V. M. Gadre, "Time- frequency localized three-band biorthogonal wavelet filter bank using semidefinite relaxation and nonlinear least squares with epileptic seizure EEG signal classification", Digital Signal Processing, Vol. 62, pp.259-273, 2017.

[23] P. Fergus, A Hussain, D. Hignett, D. AlJumeily, K. Abdel-Aziz, and H. Hamdan, "A machine learning system for automated wholebrain seizure detection", Applied Computing and Informatics, Vol. 12, pp.70-89, 2016.

[24] Y. Ma, X. Ding, Q. She, Z.Luo, T. Potter, and Y. Zhang, "Classification of motor imagery EEG signals with support vector machines and particle swarm optimization", Computational and Mathematical Methods in Medicine, Vol. 2016, 2016.

[25] P. Fergus, D. Hignett, A. Hussain, D. AlJumeily, and K. Abdel-Aziz, "Automatic epileptic seizure detection using scalp EEG and advanced artificial intelligence techniques", BioMed Research International, Vol. 2015, 2015.

[26] T. S. Kumar, V. Kanhangad, and R. B. Pachori, "Classification of seizure and seizure-free EEG signals using local binary patterns", Biomedical Signal Processing and Control, Vol. 15, pp. 33-40, 2015.

[27] A. X. Stewart, A. Nuthmann, and G. Sanguinetti, "Single-trial classification of EEG in a visual object task using ICA and machine learning", Journal of Neuroscience Methods, Vol.228, pp.1-14, 2014.

[28] Y. Kumar, M. L. Dewal, and R. S. Anand, "Epileptic seizure detection using DWT based fuzzy approximate entropy and support vector machine", Neurocomputing, Vol. 133, pp.271279, 2014.

[29] V. Joshi, R. B. Pachoriand, and A. Vijesh, "Classificatin of ictal and seizure-free EEG signals using fractional linear prediction", Biomedical Signal Processing and Control, Vol. 9, pp.1-5, 2014.

[30] K. C. Hsu and S. N. Yu, "Detection of seizures in EEG using subband nonlinear parameters and genetic algorithm", Computers in Biology and Medicine, Vol. 40, pp.823-830. 2010.

[31] G. R. Bermúdez, P. J. G.Laencina, J. R. González, and J. R.Dorda, "Efficient feature selection and linear discrimination of EEG signals", Neurocomputing, Vol. 115, pp.161165, 2013. 
[32] J. Rasekhi, M. R. Mollaei, M.Bandarabadi, C. A. Teixeira, and A. Dourado, "Preprocessing effects of 22 linear univariate features on the performance of seizure prediction methods", Journal of Neuroscience Methods, Vol. 217, pp. 9-16, 2013.

[33] D. of Epileptology University of Bonn, "EEG time series data", Department of Epileptology University of Bonn, 2016.

[34] M. A. Hall Ian H. Witten, E. Frank, "Data Mining: Practical Machine Learning Tools and Techniques (Third Edition)", The Morgan Kaufmann Series in Data Management Systems, Morgan Kaufmann, Boston, third edition ed, 2011.

[35] U. R. Acharya, F. Molinari, S V. Sree, S. Chattopadhyay, K.H. Ng, and J. S Suri, "Automated diagnosis of epileptic EEG using entropies", Biomedical Signal Processing and Control, Vol. 7 , pp.401-408, 2012.

[36] N. Nicolaou and J. Georgiou, "Detection of epileptic electroencephalogram based on permutation entropy and support vector machines", Expert Systems with Applications, Vol. 39, pp. 202-209, 2012.

[37] R. B. Pachori and S. Patidar, "Epileptic seizure classification in EEG signals using secondorder difference plot of intrinsic mode functions", Computer Methods and Programs in Biomedicine, Vol. 113, pp.494-502.2015.

[38] R. J. Martis, C. Chakraborty, and A. K. Ray, "An integrated ECG feature extraction scheme using PCA and wavelet transform", In: Proc. of the 2009 Annual India Conference, pp. 1-4, 2009. 